\title{
Neutral bremsstrahlung and excimer electroluminescence in noble gases and its relevance to two-phase dark matter detectors
}

\author{
E. Borisova $^{1,2}$, A. Buzulutskov ${ }^{1,2, \mathrm{a}}(\mathbb{D}$ \\ ${ }^{1}$ Budker Institute of Nuclear Physics SB RAS, Lavrentiev Avenue 11, 630090 Novosibirsk, Russia \\ ${ }^{2}$ Novosibirsk State University, Pirogov Street 2, 630090 Novosibirsk, Russia
}

Received: 13 July 2021 / Accepted: 6 December 2021 / Published online: 23 December 2021

(C) The Author(s) 2021

\begin{abstract}
Proportional electroluminescence (EL) is the physical effect used in two-phase detectors for dark matter searches, to optically record (in the gas phase) the ionization signal produced by particle scattering in the liquid phase. In our previous work the presence of a new EL mechanism, namely that of neutral bremsstrahlung (NBrS), was demonstrated in two-phase argon detectors both theoretically and experimentally, in addition to the ordinary EL mechanism due to excimer emission. In this work the similar theoretical approach is applied to all noble gases, i.e. overall to helium, neon, argon, krypton and xenon, to calculate the EL yields and spectra both for NBrS and excimer EL. The relevance of the results obtained to the development of two-phase dark matter detectors is discussed.
\end{abstract}

\section{Introduction}

In two-phase detectors for dark matter searches and lowenergy neutrino experiments, the scattered particle produces two types of signals $[1,2]$ : that of primary scintillation, produced in the liquid and recorded promptly ("S1"), and that of primary ionization, produced in the liquid and recorded with a delay in the gas phase ("S2"). To record the S2 signal, proportional electroluminescence (EL) is used, produced by drifting electrons under high enough electric fields.

According to modern concepts [3], there are three mechanisms responsible for proportional EL in noble gases: that of excimer (e.g. $\mathrm{Ar}_{2}^{*}$ ) emission in the vacuum ultraviolet (VUV), that of emission due to atomic transitions in the near infrared (NIR), and that of neutral bremsstrahlung (NBrS) emission in the UV, visible and NIR range. In the following these three mechanisms are referred to as excimer (or else ordinary) EL, atomic EL and NBrS EL, respectively.

\footnotetext{
a e-mail: A.F.Buzulutskov@inp.nsk.su (corresponding author)
}

The presence of NBrS EL in two-phase Ar detectors has for the first time been demonstrated in our previous work [4], both theoretically and experimentally. In particular it was shown that the NBrS effect can explain two intriguing observations in EL radiation of gaseous Ar: that of the substantial contribution of the non-VUV spectral component (in the UV, visible and NIR range), and that of the photon emission at lower electric fields, below the Ar excitation threshold. The merit of that work was that it transformed the idea of $\mathrm{NBrS}$ EL from a hypothesis [5] into a quantitative theory [4]. The success of the NBrS theory developed there was that it correctly predicted the absolute value of the EL yield below the Ar excitation threshold.

In this work, the similar theoretical approach is applied to all noble gases both for $\mathrm{NBrS}$ and excimer EL, to calculate the EL yields and spectra: to $\mathrm{He}, \mathrm{Ne}, \mathrm{Ar}, \mathrm{Kr}$ and $\mathrm{Xe}$. The relevance of the results obtained to the development of twophase dark matter detectors is also discussed.

\section{Overview of electroluminescence (EL) mechanisms in noble gases}

The three EL mechanisms in noble gases are illustrated in Figs. 1 and 2, on the example of Ar, showing the dependence of the reduced EL yield on the reduced electric field. The first figure shows the experimentally measured yields, while the second one compares the experimental yields to those calculated theoretically.

Here the reduced EL yield, $Y_{E L} / N$, is defined as the number of photons produced per unit drift path $\left(d N_{p h} / d x\right)$ and per drifting electron, normalized to the atomic density $(N)$ :

$\frac{Y_{E L}}{N}=\frac{d N_{p h}}{d x N N_{e} d V}$,

where $N_{e}$ is the density of drifting electrons, $d V$ is the volume and $N_{e} d V$ is thus the number of drifting electrons. The 


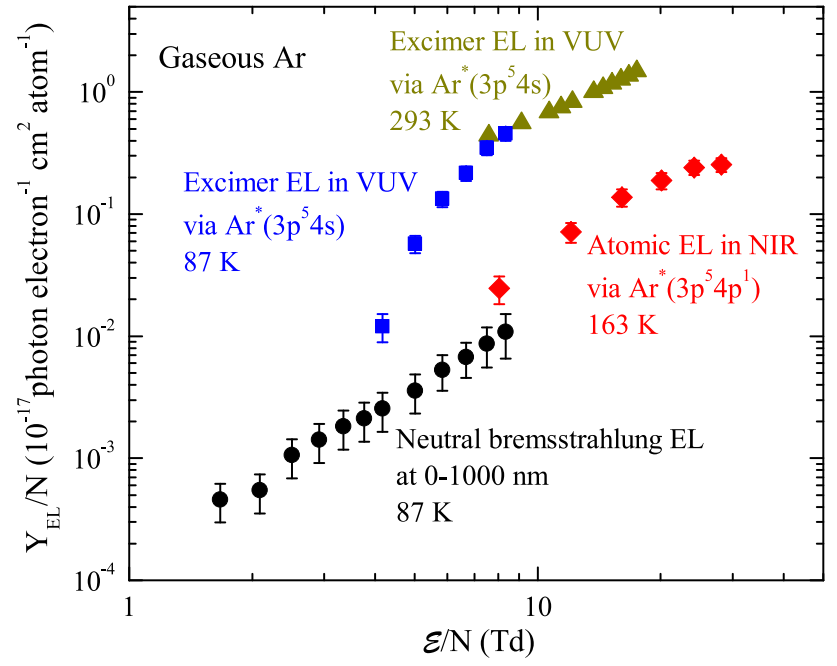

Fig. 1 Summary of experimental data on reduced electroluminescence (EL) yield in gaseous Ar for all known EL mechanisms: for neutral bremsstrahlung $(\mathrm{NBrS}) \mathrm{EL}$ at wavelengths of $0-1000 \mathrm{~nm}$ obtained at $87 \mathrm{~K}$ in $[4,6]$; for excimer EL in the VUV going via $\mathrm{Ar}^{*}\left(3 p^{5} 4 s\right)$ excited states, obtained at $87 \mathrm{~K}$ in [6] and at $293 \mathrm{~K}$ in [7] (the data points of the latter below $7 \mathrm{Td}$ are not shown as they include both excimer and $\mathrm{NBrS}$ EL); for EL in the NIR, going via $\operatorname{Ar}^{*}\left(3 p^{5} 4 p\right)$ excited sates, obtained at $163 \mathrm{~K}$ in [8]. For $\mathrm{NBrS}$ EL at $\mathcal{E} / N>4 \mathrm{Td}$, the experimental EL yield exceeds that predicted by the theory for standard NBrS EL; therefore the data points at these fields might be somewhat incorrect, as they were obtained assuming the standard NBrS EL emission spectrum which might not be the case

reduced electric field is defined as $\mathcal{E} / N$, where $\mathcal{E}$ is the electric field. It is expressed in Td units: $1 \mathrm{Td}=10^{-17} \mathrm{~V} \mathrm{~cm}$, corresponding to the electric field of $0.87 \mathrm{kV} / \mathrm{cm}$ in gaseous $\mathrm{Ar}$ in the two-phase mode at $87.3 \mathrm{~K}$ and $1.00 \mathrm{~atm}$.

Excimer (ordinary) EL is due to emission of noble gas excimers, $\operatorname{Ar}_{2}^{*}\left({ }^{1,3} \Sigma_{u}^{+}\right)$, produced in three-body atomic collisions of excited atoms, $\operatorname{Ar}^{*}\left(3 p^{5} 4 s\right)$, which in turn are produced by drifting electrons in electron-atom collisions (see reviews $[1-3,10])$ :

$$
\begin{aligned}
& e^{-}+\operatorname{Ar} \rightarrow e^{-}+\operatorname{Ar}^{*}\left(3 p^{5} 4 s\right), \\
& \operatorname{Ar}^{*}\left(3 p^{5} 4 s\right)+2 \operatorname{Ar} \rightarrow \operatorname{Ar}_{2}^{*}\left({ }^{1,3} \Sigma_{u}^{+}\right)+\operatorname{Ar}, \\
& \operatorname{Ar}_{2}^{*}\left({ }^{1,3} \Sigma_{u}^{+}\right) \rightarrow 2 \operatorname{Ar}+h v .
\end{aligned}
$$

Excimer EL in noble gases has a threshold in the electric field (of about $4 \mathrm{Td}$ for Ar), defined by the lowest atomic excitation levels $\mathrm{Ar}^{*}\left(3 p^{5} 4 s\right)$. In addition it is characterized by emission continuum in the VUV (so-called "second continuum" [11]): Fig. 3 shows this for all noble gases. From this figure one can see that excimer EL can be recorded directly only in Xe, using photomultiplier tubes (PMTs) or silicon photomultipliers (SiPMs) with quartz windows, while in other noble gases ( $\mathrm{He}, \mathrm{Ne}, \mathrm{Ar}$ and $\mathrm{Kr}$ ) it can be recorded indirectly (since quartz is not transparent below $160 \mathrm{~nm}$ ) using a wavelength shifter (WLS), typically tetraphenyl-butadiene (TPB) [12].

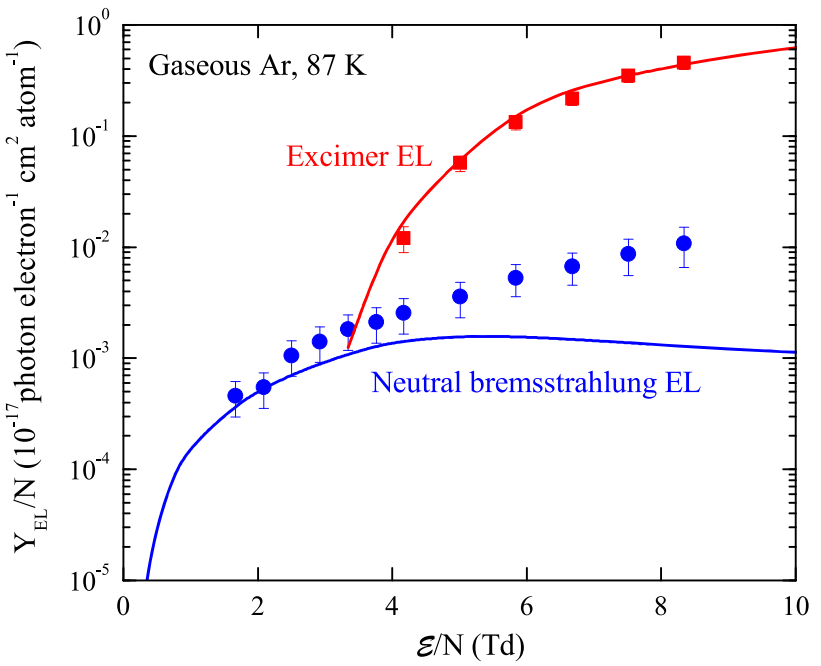

Fig. 2 Experimental data (points) on reduced EL yield in gaseous Ar at lower reduced electric fields, below $9 \mathrm{Td}$, for $\mathrm{NBrS}$ EL $[4,6]$ and excimer EL [6], in comparison with the theory (curves) presented in [4] and [9] respectively. For $\mathrm{NBrS}$ EL at $\mathcal{E} / N>4 \mathrm{Td}$, the experimental EL yield exceeds that predicted by the theory for standard NBrS EL; therefore the data points at these fields might be somewhat incorrect, as they were obtained assuming the standard NBrS EL emission spectrum which might not be the case

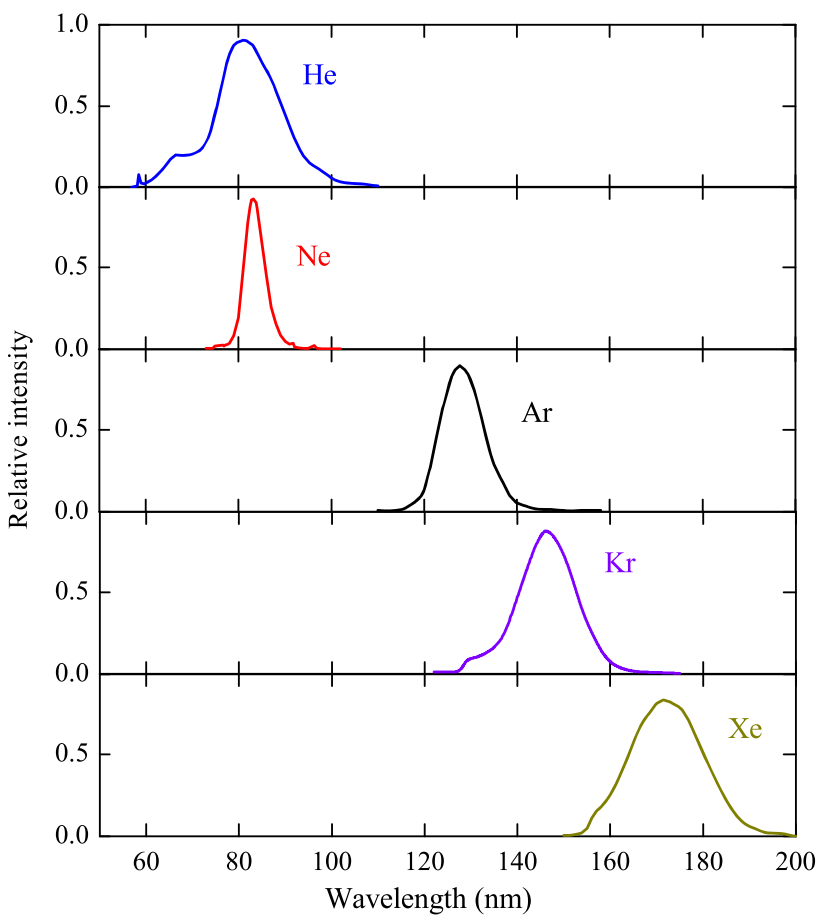

Fig. 3 Spectra of the second VUV continuum in gaseous $\mathrm{He}, \mathrm{Ne}, \mathrm{Ar}$, $\mathrm{Kr}$ and $\mathrm{Xe}$ due to excimer emission. In He it was induced by condensed discharge at a pressure of 40 Torr [13,14], while in $\mathrm{Ne}, \mathrm{Ar}, \mathrm{Kr}$ and $\mathrm{Xe}$ it was induced by electron beam at a pressure of $120 \mathrm{kPa}$ [15]

Atomic EL in the NIR is due to atomic transitions between the excited states $[8,19]$ :

$\operatorname{Ar}^{*}\left(3 p^{5} 4 p\right) \rightarrow \operatorname{Ar}^{*}\left(3 p^{5} 4 s\right)+h v$ 


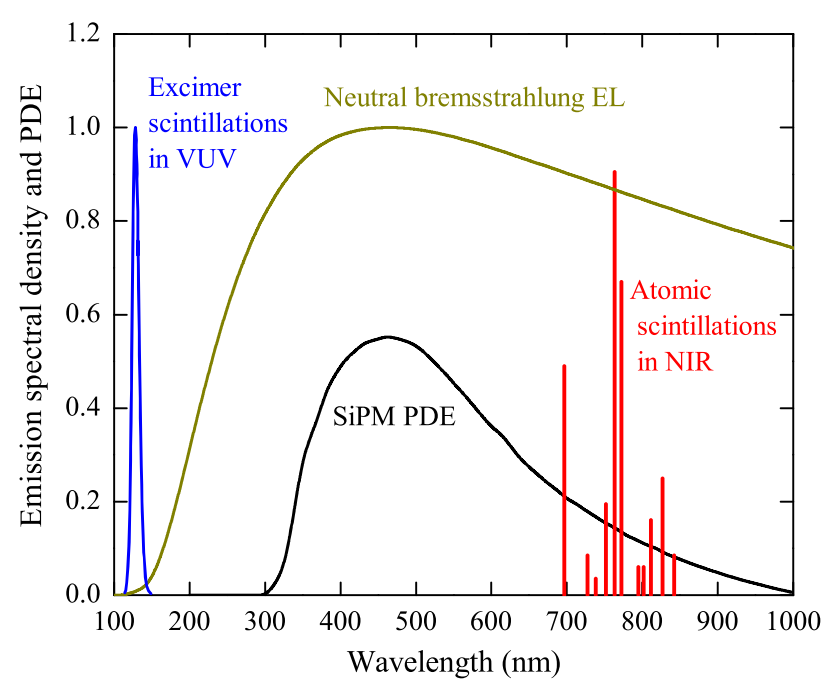

Fig. 4 Photon emission spectra in gaseous Ar due to excimer scintillations in the VUV measured in [15], NBrS EL at $8.3 \mathrm{Td}$ theoretically calculated in [4] and atomic scintillations in the NIR measured in $[16,17]$. Also shown is the photon detection efficiency (PDE) of the typical SiPM [18]

It has a line emission spectrum $[3,16]$ (see Fig. 4) and a $\sim 1$ Td higher electric field threshold compared to excimer EL, i.e. at about $5 \mathrm{Td}$ [19].

Neutral bremsstrahlung (NBrS) EL is due to bremsstrahlung of drifting electrons scattered (elastically or inelastically) on neutral atoms:

$$
\begin{aligned}
& e^{-}+\mathrm{Ar} \rightarrow e^{-}+\mathrm{Ar}+h v, \\
& e^{-}+\mathrm{Ar} \rightarrow e^{-}+\mathrm{Ar}^{*}+h v .
\end{aligned}
$$

In the most elaborated way, NBrS EL was introduced in [4] applying both theoretical and experimental approach to explain two observations: the photon emission below the $\mathrm{Ar}$ excitation threshold and the contribution of the non-VUV spectral component in proportional EL (extending from the UV to NIR). It was further experimentally studied in $[6,20-$ 24].

In addition, the practical application of NBrS EL in twophase Ar detectors has been recently demonstrated by the DarkSide-20k collaboration [18], using direct SiPM-matrix readout of the EL gap in the visible range.

The theory predicts that the contribution of NBrS EL due to elastic collisions (reaction 4) is considerably larger than that of inelastic [4], since the elastic cross section for electron-atom collision is considerably larger than that of inelastic. This should be true for all noble gases. Accordingly, in this work we consider NBrS EL due to elastic collisions only.

NBrS EL has a continuous emission spectrum, extending from the UV to the visible and NIR range: see Fig. 4. From Figs. 1 and 2 one can see that NBrS EL, albeit being significantly weaker than excimer EL above the Ar excitation threshold, has no threshold in electric field, in contrast to excimer EL, and thus dominates below the threshold.

At lower electric fields, below $4 \mathrm{Td}$ corresponding to $\mathrm{Ar}$ excitation threshold, the NBrS theory developed in [4] correctly predicts the absolute value of the EL yield. This is seen when comparing the experimental and theoretical EL yields in Fig. 2 and when comparing the experimental and theoretical photon emission spectra [4,20,24].

On the other hand, at higher fields, above $4 \mathrm{Td}$, the experimental EL yield quickly diverges from that of the theory [4], exceeding that in the UV spectral range (below $400 \mathrm{~nm}$ ) [20]. In [4] this excess of experimental data over theoretical prediction was proposed to be explained by the contribution of electron scattering on sub-excitation Feshbach resonances [25] (going via intermediate negative ion states $\mathrm{Ar}^{-}\left(3 p^{5} 4 s^{2}\right)$ ), which might be accompanied by enhanced photon emission $[4,26]$ :

$e^{-}+\mathrm{Ar} \rightarrow \mathrm{Ar}^{-}\left(3 p^{5} 4 s^{2}\right) \rightarrow e^{-}+\mathrm{Ar}+h v$.

There are many negative ion resonances above the Ar excitation threshold other than those of Feshbach [25], which may also give rise to photon emission similar to Eq. (6). The energies of Feshbach resonances are close to that of the lowest excitation level (see Table 1, item 4 and 5), which should result in a field dependence similar to that of excimer EL, namely in the linear growth of the photon yield with the electric field started at almost the same thresold as that of excimer EL (i.e. at 4 Td). Since the photon emission spectrum of this mechanism can differ from that of "standard" NBrS EL, the experimental data points in Figs. 1 and 2 at $\mathcal{E} / N>4$ Td might be somewhat incorrect, as they were obtained assuming the standard NBrS EL emission spectrum which might not be the case. Note that sub-excitation Feshbach resonances exist in all noble gases: see Table 1 (item 4) for their description and energies. Thus this mechanism (neutral bremsstrahlung on resonances) can be applied to all noble gases, if any.

An alternative explanation of the observed excess in EL yield was considered in [24], using experimental data on the EL spectra in the visible range in pure Ar and its mixtures with $\mathrm{N}_{2}$ obtained at room temperature: the idea that emission of $\mathrm{N}_{2}$ impurity might be responsible for this excess was considered. In particular, it was observed that at a $\mathrm{N}_{2}$ content of $100 \mathrm{ppm}$ there was a significant contribution of characteristic emission peaks of $\mathrm{N}_{2}^{*}(C)$ excited states (in the range of 300-400 nm [10]), which enhanced the overall photon yield in the range of 300-600 $\mathrm{nm}$ by about a factor of 2 compared to pure $\mathrm{Ar}$ (at $8 \mathrm{Td}$ ). However, for lower $\mathrm{N}_{2}$ content, of 10 ppm, the spectrum almost did not differ from that of pure Ar. Since the claimed $\mathrm{N}_{2}$ impurity content in the experimental data of Fig. 2 was below 1 ppm [4,6], it is difficult to explain the discussed excess by the results of [24], even if to involve the hypothetical mechanism of the enhancement of $\mathrm{N}_{2}^{*}(C)$ production at low temperatures proposed elsewhere 
1128 Page 4 of 14

Eur. Phys. J. C (2021) 81:1128

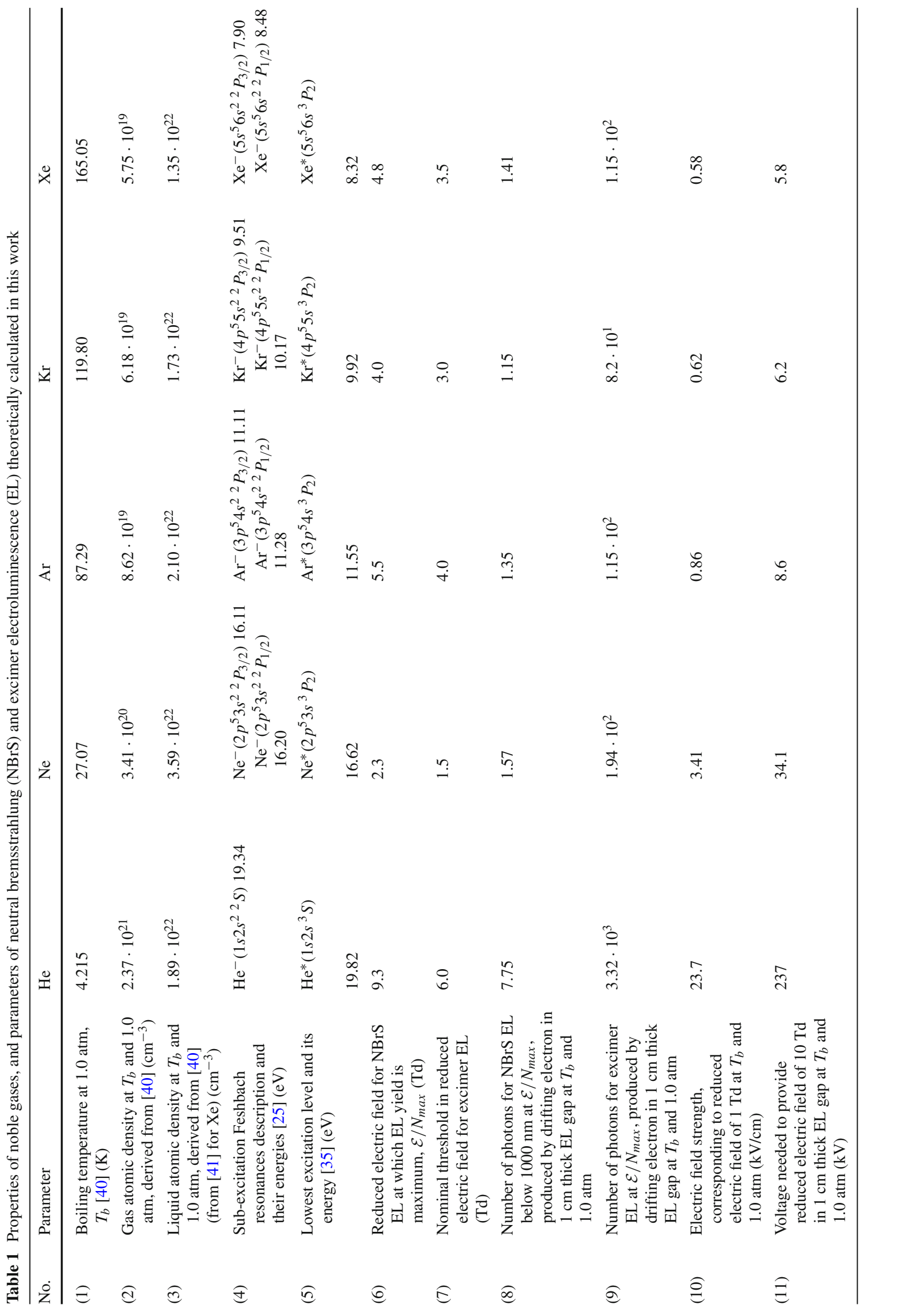

Springer 
[10]. Obviously, further research is needed to clarify this issue.

$\mathrm{NBrS}$ EL is universal in nature: it must be present in all noble gases. In particular, just recently the observation of $\mathrm{NBrS}$ EL in Xe and $\mathrm{Kr}$ below the atomic excitation threshold has been reported [27]. In the following sections we apply the theoretical method developed in [4] for Ar to all other noble gases, i.e. overall to $\mathrm{He}, \mathrm{Ne}, \mathrm{Ar}, \mathrm{Kr}$ and $\mathrm{Xe}$, both for $\mathrm{NBrS}$ and excimer EL.

\section{Theoretical formulas}

The differential cross section for NBrS photon emission is expressed via electron-atom elastic cross section $\left(\sigma_{e l}(E)\right)$ [4,28-32]:

$$
\begin{aligned}
& \frac{d \sigma}{d v}=\frac{8}{3} \frac{r_{e}}{c} \frac{1}{h v}\left(\frac{E-h v}{E}\right)^{1 / 2} \\
& \quad \times\left[(E-h v) \sigma_{e l}(E)+E \sigma_{e l}(E-h v)\right],
\end{aligned}
$$

where $r_{e}=e^{2} / m_{e} c^{2}$ is the classical electron radius, $c=v \lambda$ is the speed of light, $E$ is the initial electron energy and $h v$ is the photon energy.

The reduced EL yield $\left(Y_{E L} / N\right)$ for $\mathrm{NBrS}$ EL can be described by the following equation [4]:

$$
\left(\frac{Y_{E L}}{N}\right)_{N B r S}=\int_{\lambda_{1}}^{\lambda_{2}} \int_{h v}^{\infty} \frac{v_{e}}{v_{d}} \frac{d \sigma}{d v} \frac{d v}{d \lambda} f(E) d E d \lambda,
$$

where $v_{e}=\sqrt{2 E / m_{e}}$ is the electron velocity of chaotic motion, $v_{d}$ is the electron drift velocity, $\lambda_{1}-\lambda_{2}$ is the sensitivity region of the photon detector, $d v / d \lambda=-c / \lambda^{2}, f(E)$ is the electron energy distribution function normalized as

$$
\int_{0}^{\infty} f(E) d E=1 .
$$

Consequently, the spectrum of the reduced EL yield is

$$
\frac{d\left(Y_{E L} / N\right)_{N B r S}}{d \lambda}=\int_{h v}^{\infty} \frac{v_{e}}{v_{d}} \frac{d \sigma}{d v} \frac{d \nu}{d \lambda} f(E) d E .
$$

The reduced EL yield $\left(Y_{E L} / N\right)$ for excimer EL can be described by the following equation [4]:

$$
\left(\frac{Y_{E L}}{N}\right)_{\text {excimer }}=\int_{E_{\text {exc }}}^{\infty} \frac{v_{e}}{v_{d}} \sigma_{\text {exc }}(E) f(E) d E,
$$

where $\sigma_{\text {exc }}(E)$ is the inelastic cross-section to produce an excited state in electron-atom collisions. Similarly to [9], it is assumed that one excited state (e.g. Ar*) produces one excimer state (e.g. $\mathrm{Ar}_{2}^{*}$ ) and that one excimer produces one VUV photon.

\section{Cross sections and electron energy distribution functions}

The electron energy distribution functions were calculated by solving the Boltzmann equation using BOLSIG+ free software $[33,34]$, at a temperature corresponding to the boiling point at $1.0 \mathrm{~atm}$ for a given noble gas (see Table 1, item 1). The electron scattering cross sections from Biagi database [35], transcribed from code Magboltz (version 8.9 for $\mathrm{Ne}$ or 8.97 for other gases) [36], were used as input data.

In particular, Fig. 5 presents the following cross sections for each noble gas: that of momentum-transfer and ionization taken from the Biagi database [35], that of excitation obtained as the sum of cross sections for all given excitation states taken from the Biagi database [35], and that of total elastic for $\mathrm{Ne}, \mathrm{Ar}, \mathrm{Kr}$, and Xe taken from the BSR database [37].

It should be remarked that there are two opinions about what elastic cross section should appear in Eq. (7): that of total elastic [29,30,32,39] or momentum transfer (transport) $[28,31,32]$. In the region of interest of electron energies (1$10 \mathrm{eV}$ ), where the contribution to NBrS cross section is maximal, the transport cross section is slightly $(\leq 25 \%)$ smaller than that of total elastic in all noble gases, resulting is that the NBrS cross section would be appropriately reduced by this small factor (below 25\%), if to use the transport cross section instead of total elastic. This reduction is far smaller than the uncertainty of the theoretical model (factor of 2). Consequently, this could not change the conclusions of the calculations. Therefore in what follows, we use the total elastic cross section for all noble gases, except He where only the data on the transport cross section are available in the Biagi database.

Figure 6 shows examples of the calculated electron energy distribution function, namely the distribution functions with a prime $\left(f^{\prime}\right)$ often used instead of $f$ and normalized as

$$
\int_{0}^{\infty} E^{1 / 2} f^{\prime}(E) d E=1 .
$$

$f^{\prime}$ is considered to be more enlightening than $f$, since in the limit of zero electric field it tends to Maxwellian distribution.

Using the electron energy distribution functions, one can calculate the electron mean velocity of directed motion (drift velocity, $\left.v_{d}\right)$ and that of chaotic motion $\left(v_{e}\right)$ for all noble gases: both are shown in Fig. 7 as a function of the reduced electric field. It is possible to check the correctness of the distribution functions by comparing the calculated and measured electron drift velocities: this is done in Fig. 8 using the experimental data compiled in [38]. It can be seen that the theoretical and experimental drift velocities are in excellent agreement, thus confirming the correctness of the calculated distribution functions for all noble gases. 

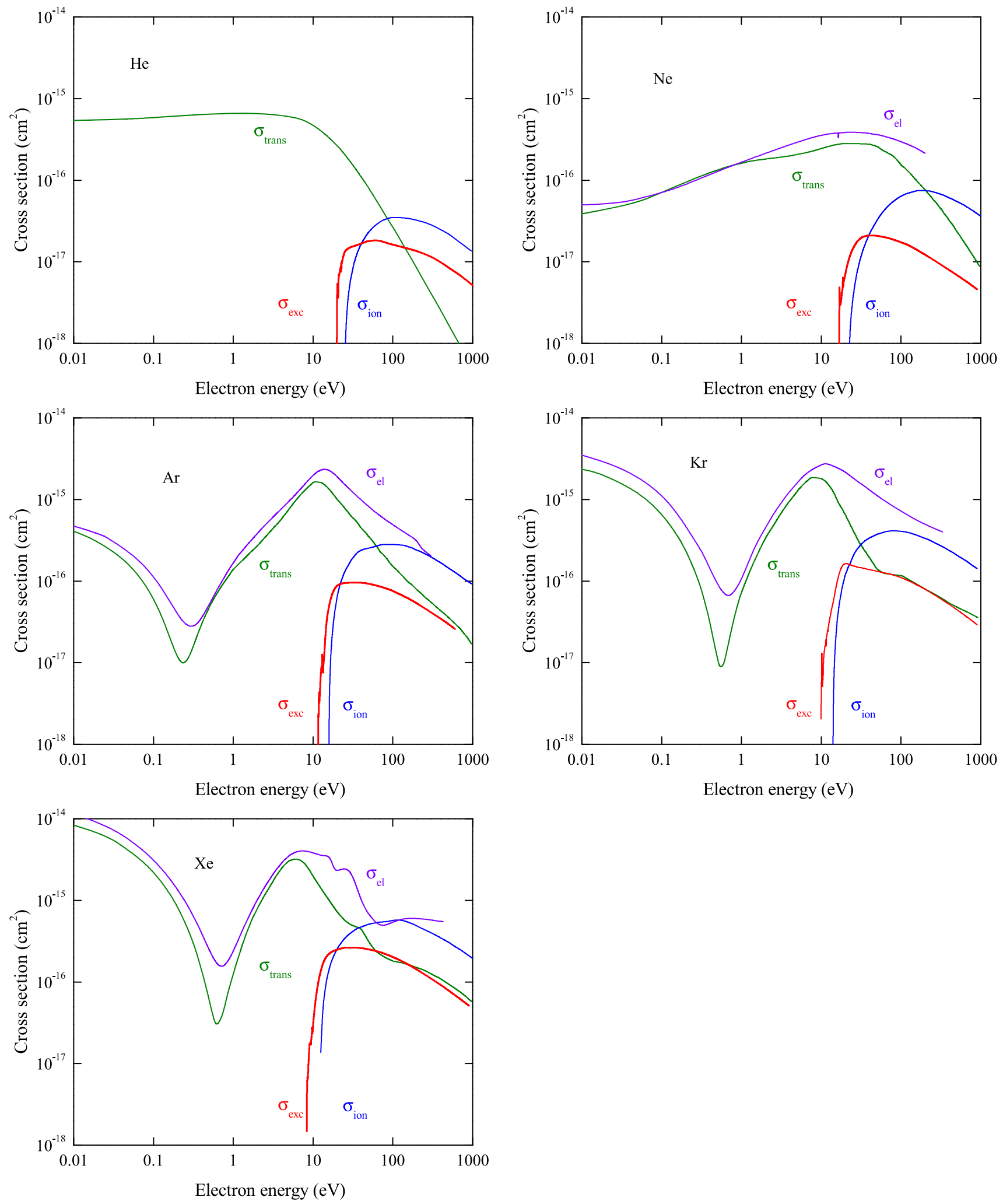

Fig. 5 Electron scattering cross section in noble gases as a function of electron energy used in this work, obtained from the data bases [35,37]: that of total elastic $\left(\sigma_{e l}\right)$, momentum transfer $\left(\sigma_{\text {trans }}\right)$, excitation $\left(\sigma_{\text {exc }}\right)$

and ionization $\left(\sigma_{i o n}\right)$. The excitation cross section was obtained as the sum of those for all given excitation states 

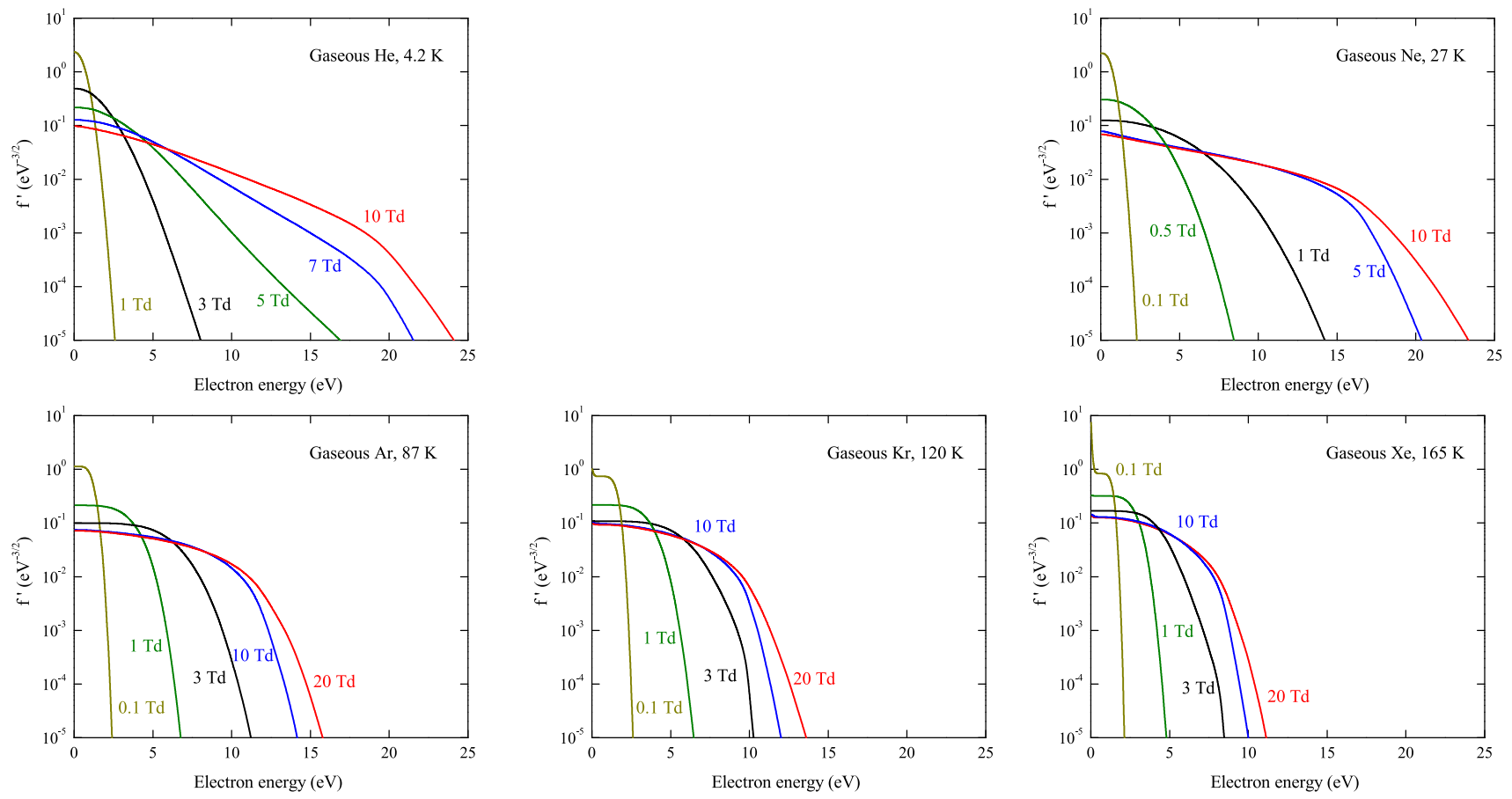

Fig. 6 Electron energy distribution functions with a prime $\left(f^{\prime}\right)$ in noble gases, normalized as in Eq. (12), calculated using Boltzmann equation solver BOLSIG+ [34], at different reduced electric fields

Electron chaotic velocity in Fig. 7 (bottom) in fact reflects the dependence of the electron mean energy on the electric field: the mean energy was calculated in a similar way and is shown in Fig. 9.

From Fig. 6 one can see that at reduced electric fields exceeding $10 \mathrm{Td}$ the distribution function tends to have a high-energy cut-off, corresponding to the onset of significant electron energy losses due to exciting the lowest atomic excitation level. The energy of the latter is lower for heavier noble gases (see Table 1, item 5) resulting in a correspondingly lower cut-off energy.

This effect also explains why at higher electric fields the electron drift velocity, chaotic velocity and energy are lower for a heavier noble gas (see Figs. 7 and 9): these quantities decrease in the series $\mathrm{Ne}, \mathrm{Ar}, \mathrm{Kr}$ and $\mathrm{Xe}$.

It is interesting that $\mathrm{He}$ violates this rule: the electron drift velocity is always lower in $\mathrm{He}$ than in $\mathrm{Ne}$, and the electron energy in $\mathrm{He}$ is lower than that of all other noble gases at reduced electric fields below $5 \mathrm{Td}$. This strangeness of $\mathrm{He}$ is fully explained by the properties of its electron elastic cross section which defines the electron transport in this energy range: see Fig. 5. Indeed, at electron energies below $3 \mathrm{eV}$, the electron elastic cross section in $\mathrm{He}$ is significantly larger than that of other noble gases, in particular due to the fact that it does not have a Ramsauer minimum in the elastic cross section, in contrast to $\mathrm{Ar}, \mathrm{Kr}$ and $\mathrm{Xe}$. The larger the elastic cross section, the lower the electron mean energy and velocity in this energy region.

It should be remarked that the distribution function and other quantities in Figs. 6, 7 and 9 (top), obtained by solving the Boltzmann equation, are actually averaged over the collisions and hence also over the time between the collisions. On the other hand, in microscopic approach [9], the energy just before the collision is used, which obviously exceeds the time-averaged energy since the electrons are accelerated by the electric field between the collisions: compare Figs. 9 (top) and 9 (bottom). In microscopic approach this energy is substituted into the formulas for the cross section, when simulating the event. This raises the question which distribution function should be used in Eqs. (8), (10) and (11): that of time-averaged, obtained by solving the Boltzmann equation, or that averaged before collisions, obtained in microscopic approach?

In our previous work [4] both distributions functions were used, and the variation of the results between them was taken to determine the theoretical uncertainty: the latter reached a factor of 2 for the EL yield. Such a theoretical uncertainty can be considered as quite acceptable for the EL yields varying by 4 orders of magnitude (see Fig. 2). For simplicity, in this work we restrict ourselves to only one type of distribution function, namely to that obtained by solving the Boltzmann equation. 

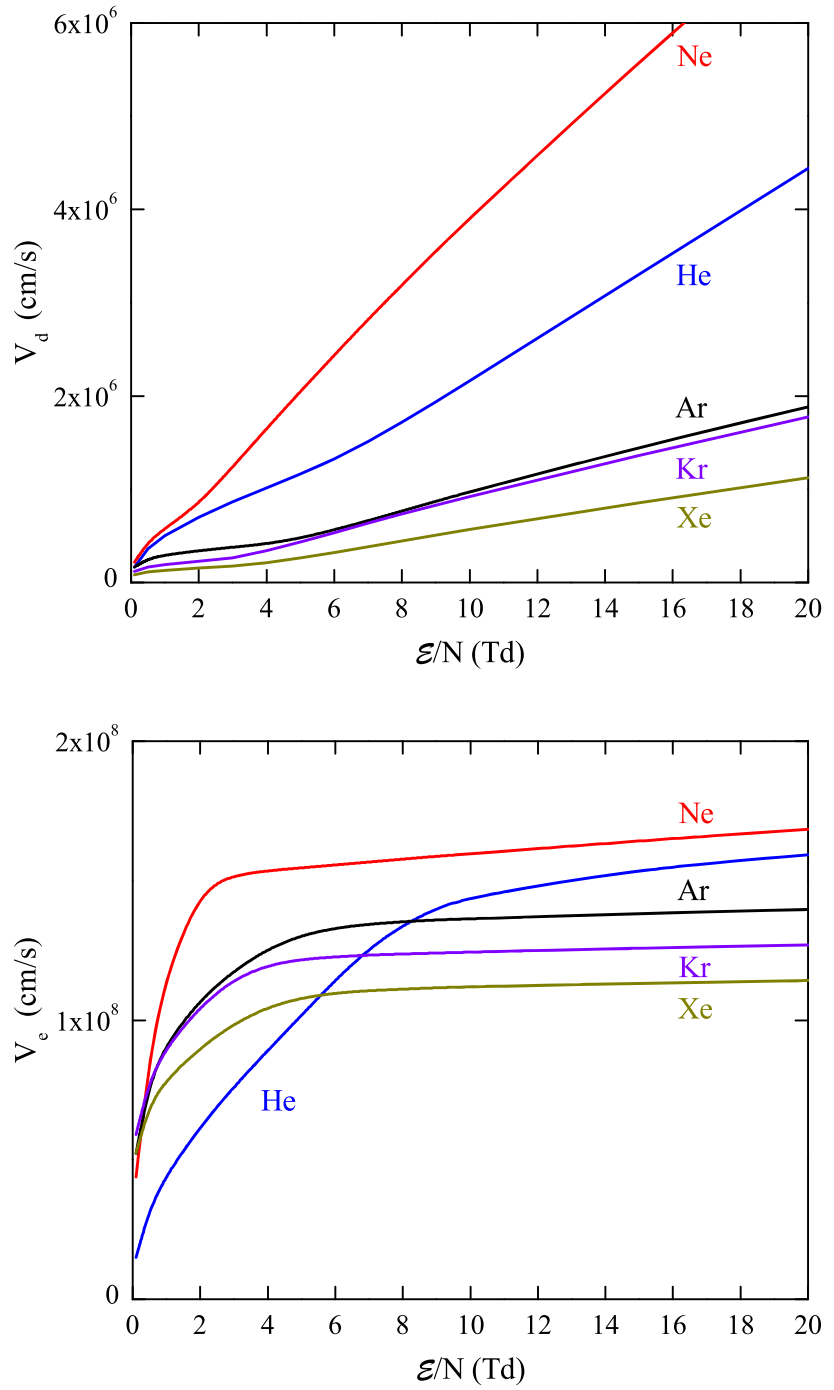

Fig. 7 Electron drift velocity (top) in noble gases and that of chaotic motion (bottom) as a function of the reduced electric field, calculated in this work using Boltzmann equation solver

\section{EL spectra and yields}

Figure 10 show the $\mathrm{NBrS}$ spectra of the reduced EL yield. The spectra were calculated by numerical integration of Eq. (10). One can compare these to the spectra of excimer luminescence shown in Fig. 3.

One can see that the NBrS EL spectra are very similar in all noble gases: they are rather flat, extending from the UV to visible and NIR range. In each noble gas, the NBrS EL spectrum has a broad maximum that gradually moves to shorter wavelengths with the electric field. The fact that the NBrS EL spectra do not differ much in noble gases is confirmed in Fig. 11, showing how the mean wavelength of the spectrum below $1000 \mathrm{~nm}$ depends on the electric field: it decreases from $700-750 \mathrm{~nm}$ at $1 \mathrm{Td}$ to $550-650 \mathrm{~nm}$ at 10 $\mathrm{Td}$, in $\mathrm{Ar}, \mathrm{Kr}$ and $\mathrm{Xe}$, and to $500 \mathrm{~nm}$, in $\mathrm{He}$ and $\mathrm{Ne}$.
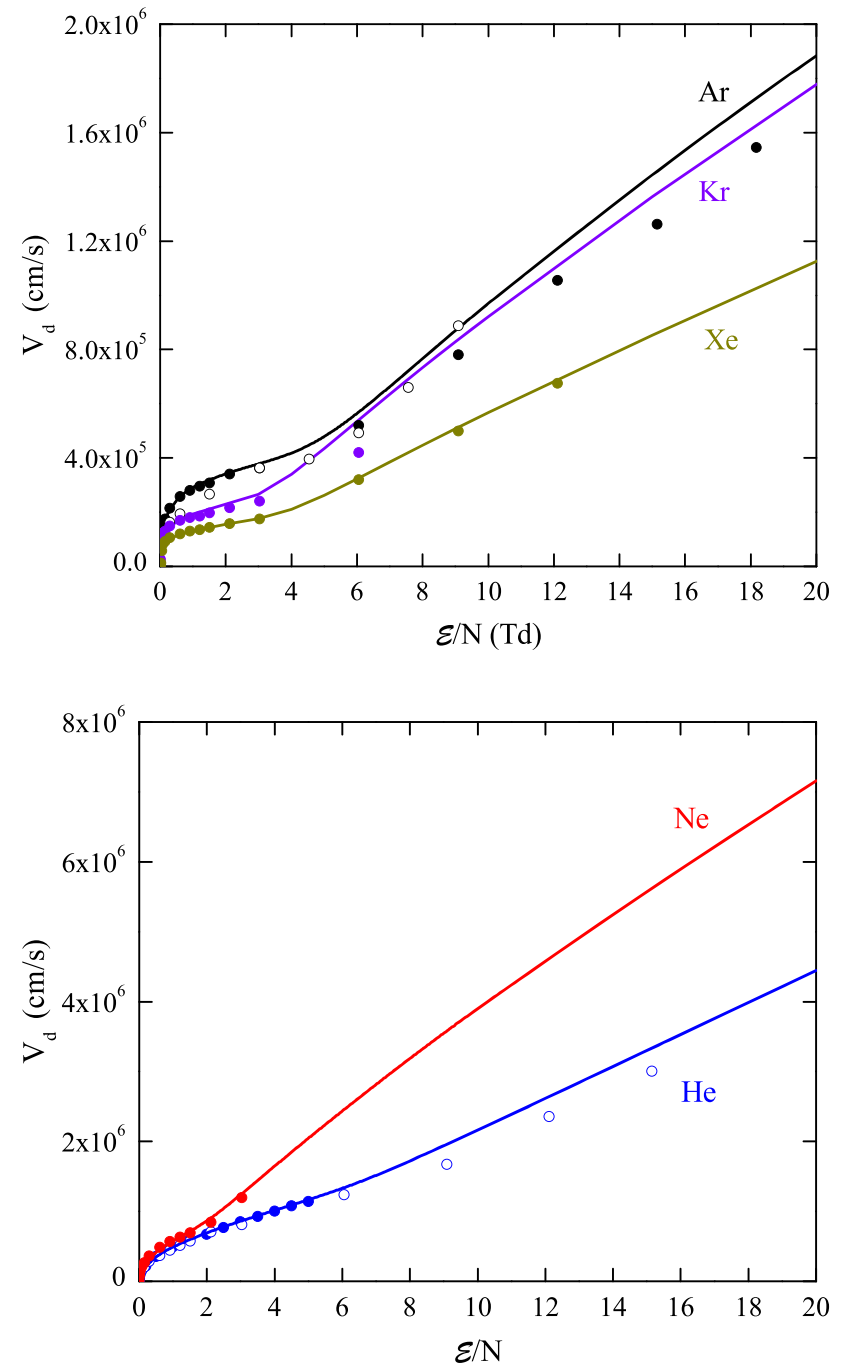

Fig. 8 Comparison of electron drift velocity $\left(v_{d}\right)$ in noble gases theoretically calculated in this work (curves) with that measured in experiment [38] (data points). The color of the curve and the data points is the same for a given gas

In all noble gases, the vast majority of the spectrum is above $200 \mathrm{~nm}$ (in the UV, visible and NIR range), i.e. just in the sensitivity region of commonly used PMTs and SiPMs. This implies a possible practical application of NBrS EL, namely the method of direct optical readout of two-phase detectors in the visible range, i.e. without using WLS. Such a technique has been recently demonstrated in two-phase Ar detector with direct SiPM-matrix readout [18].

The EL yields for both NBrS and excimer EL are presented in Figs. 12, 13 and 14, obtained by numerical integration of Eqs. (8) and (11). One can see from Fig. 12 that the ratio between NBrS and excimer EL yields and their field dependence are generally the same for all noble gases. Details are described below.

Figures 12 and 13 shows the reduced EL yield of NBrS EL as a function of the reduced electric field, obtained by 

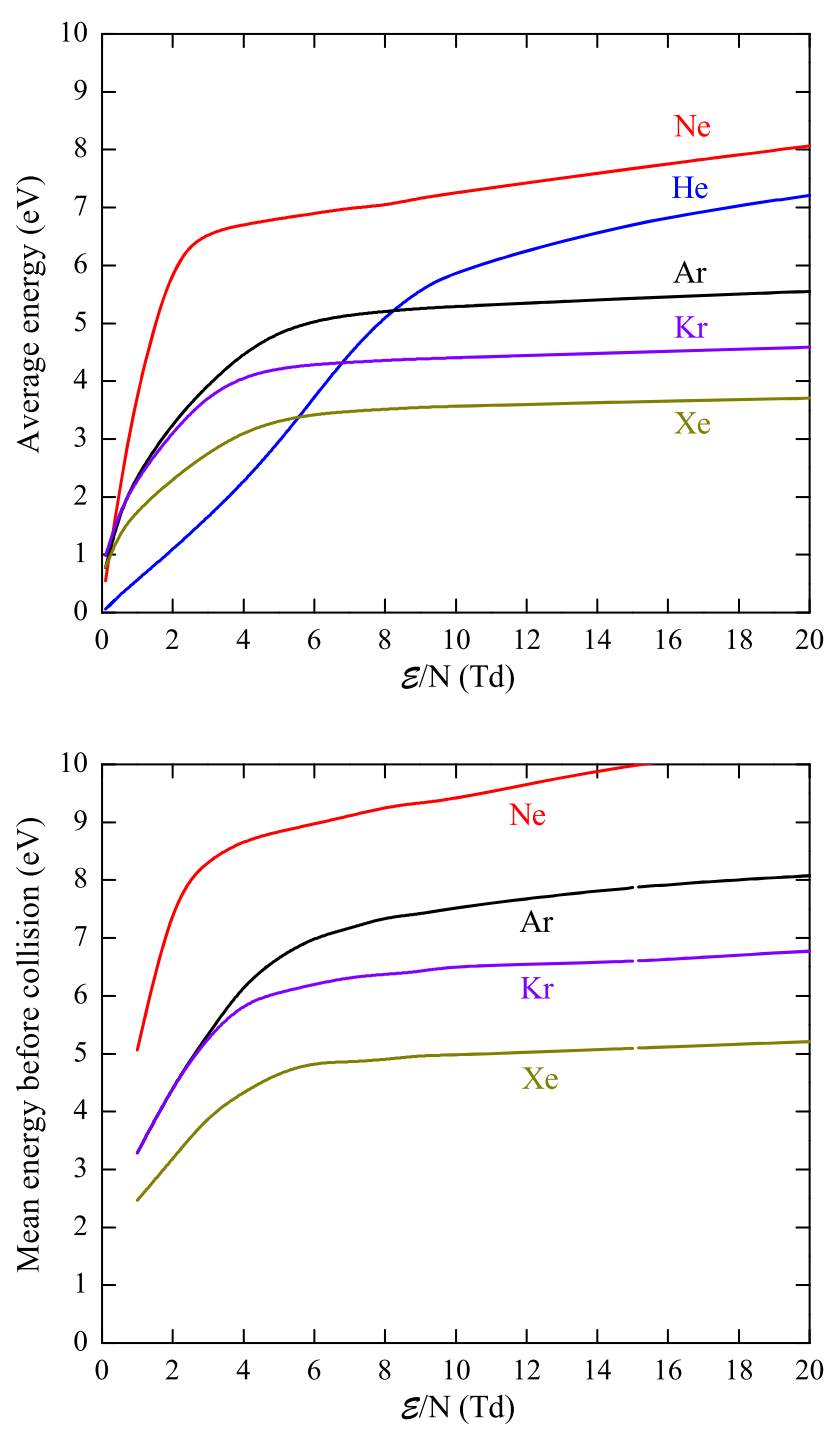

Fig. 9 Electron mean energy as a function of the reduced electric field, calculated in this work using Boltzmann equation solver (top). Also shown is the mean energy reached by the electrons before collisions, taken from [9] where a microscopic approach was used for electron transport simulation (bottom)

integration over the wavelength range of $0-1000 \mathrm{~nm}$. The long-wavelength limit of $1000 \mathrm{~nm}$ is defined by that of SiPM sensitivity (see Fig. 4). Thereby this yield corresponds to the maximum number of $\mathrm{NBrS}$ photons that can ever be detected by existing devices.

One can see from Figs. 12 and 13 that the NBrS EL yield first increases with the electric field, reaches a maximum at a certain field value $\left(\mathcal{E} / N_{\max }\right.$; see Table 1 , item 6$)$ and then slowly decreases. Such a behavior, at a slowly varying elastic cross section (shown in Fig. 5), reflects that of the $v_{e} / v_{d}$ ratio in Eq. (8): see Fig. 7. In terms of reduced EL yield, heavy noble gases are superior to light noble gases: the reduced yield of NBrS EL in $\mathrm{Ar}, \mathrm{Kr}$ and $\mathrm{Xe}$ at maximum can exceed that of $\mathrm{He}$ and Ne by a factor of 5 (see Fig. 13).

It should be remarked that the present theory of $\mathrm{NBrS}$ EL cannot explain the excess at higher fields of experimentally measured EL yields over theoretical prediction $[4,20]$ : see Fig. 2. In Ar, the excess factor reaches 10 at a reduced field of $8 \mathrm{Td}$. As discussed in Sect. 2, this excess might be explained by the additional EL mechanism, namely by $\mathrm{NBrS}$ on Feshbach and other negative ion resonances (see Eq. (6)), which is not accounted for by the present theory. Since Feshbach resonances exist in all noble gases (see Table 1, item 4), such an excess $\mathrm{NBrS}$ emission might exist in all noble gases as well. Further research is needed to clarify this issue.

The reduced EL yield of excimer EL is shown in Figs. 12 and 14 for all noble gases. One can see that the yield in the first approximation increases linearly with the field. That is why excimer EL is called proportional.

In addition, excimer EL has a well-defined threshold in the electric field, in contrast to NBrS EL the threshold of which tends to zero: compare Figs. 13 and 14. The "nominal" excimer EL threshold can be defined as the intersection of extrapolation of the linear part of the curve with horizontal axis. The resulted thresholds are presented in Table 1 (item 7); these amount to 6.0, 1.5, 4.0, 3.0 and 3.5 $\mathrm{Td}$ for $\mathrm{He}, \mathrm{Ne}$, $\mathrm{Ar}, \mathrm{Kr}$ and $\mathrm{Xe}$ respectively, in reasonable agreement with those obtained in microscopic approach [9]. It is interesting that due to interplay between the elastic and excitation cross sections, the minimum and maximum thresholds belong to $\mathrm{Ne}$ and He respectively.

For a reduced electric field of $10 \mathrm{Td}$, the reduced yield for excimer EL increases in the serious $\mathrm{He}, \mathrm{Ne}, \mathrm{Ar}, \mathrm{Kr}$ and $\mathrm{Xe}$, the difference between $\mathrm{He}$ and Xe reaching a factor of 5 (see Fig. 14). On the other hand, the reduced yield in Ne becomes equal to that of $\mathrm{Ar}$ at $8 \mathrm{Td}$ and to that of $\mathrm{Kr}$ and $\mathrm{Xe}$ at $5 \mathrm{Td}$. This is because Ne has the lowest excimer EL threshold, of $1.5 \mathrm{Td}$.

The ratio between the excimer and the NBrS EL yield can be deduced from Fig. 12: in each noble gas, it changes from about 1 at the nominal threshold of excimer EL to about 1000 at $10 \mathrm{Td}$. The latter might be reduced to about 100 if to take into account the $\mathrm{NBrS}$ enhancement at higher fields observed in experiment (see above).

The reliability of the results obtained in this work using the approach of Boltzmann equation solver can be checked by comparing to the calculations in the microscopic approach [9]. This comparison is done in Fig. 12 for excimer EL yields in all noble gases, except He: there is a rather good compliance between the two approaches. The discrepancy at higher fields in $\mathrm{Ne}$ and $\mathrm{Ar}$ is due to the multiplication of electrons by impact ionization: in our approach we consider the EL yield per drifting electron, while in the microscopic approach the yield was taken for all electrons. 

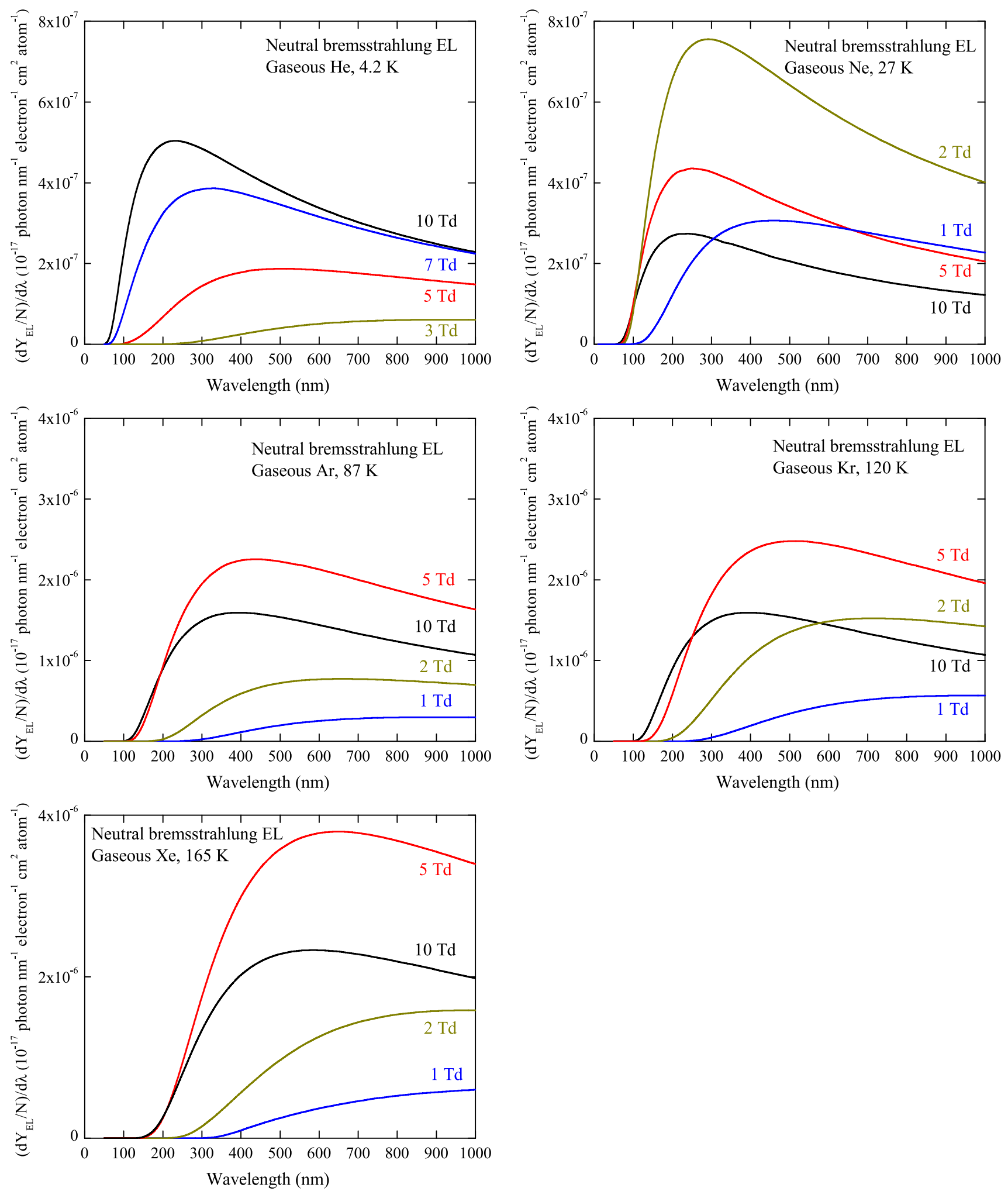

Fig. 10 Spectra of NBrS EL yield in noble gases at different reduced electric fields, calculated using Eq. (10) 


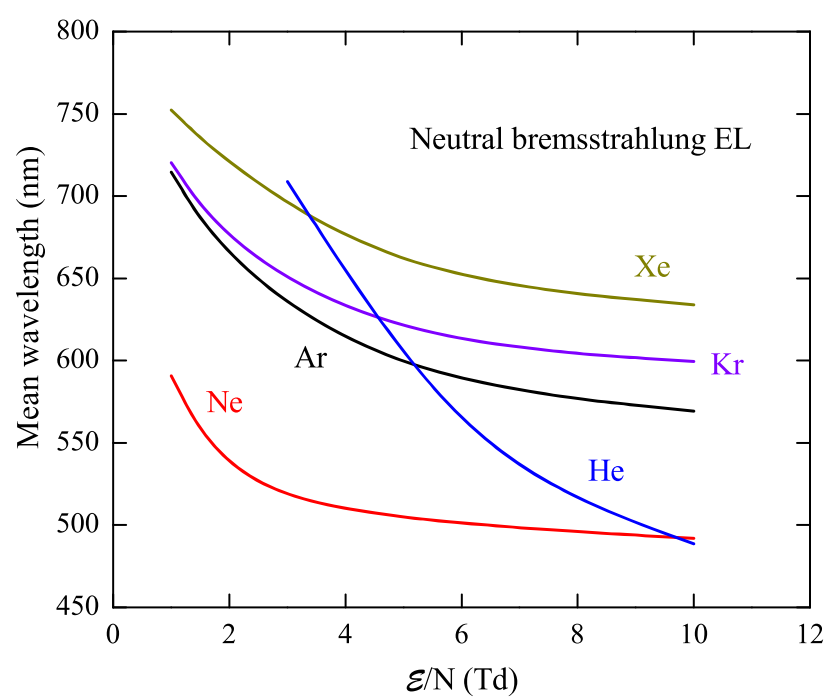

Fig. 11 Mean wavelength of NBrS EL below $1000 \mathrm{~nm}$ in noble gases as a function of the reduced electric field

\section{Relevance to two-phase dark matter detectors}

In this section the relevance of the results obtained to twophase detectors for dark matter search is discussed. We evaluate here the absolute EL yields, in terms of the number of photons produced by a drifting electron, and absolute electric fields and voltages needed to provide given reduced electric fields: these are presented in Table 1 (items 8-11). The estimations are given for a particular EL gap of a thickness of $1 \mathrm{~cm}$, used in practice in two-phase Ar and Xe detectors $[1,42,43]$.

Using gas atomic densities of Table 1 (item 2), it can be deduced that the typical reduced electric fields in the EL gap, in dark matter search experiments, were 7-10 Td in twophase Xe detectors $[1,42,43]$ and $4.6 \mathrm{Td}$ in two-phase $\mathrm{Ar}$ detector [44]. Higher reduced fields in two-phase Xe detectors are explained by the necessity to operate at higher extraction fields at the liquid-gas interface to provide the effective electron transmission through it [1].

The reduced electric field at which NBrS EL yield has a maximum predicted by the theory, $\mathcal{E} / N_{\max }$, is about $1-1.5 \mathrm{Td}$ higher than the threshold of excimer EL: see Table 1 (items 6 and 7). Therefore it is natural to compare the EL yields at just $\mathcal{E} / N_{\max }$ where both EL mechanisms exist: see Table 1 (items 8 and 9). At such fields, the number of photons of NBrS EL in the two-phase mode is $1.2-1.6$ per drifting electron per $1 \mathrm{~cm}$ in all noble gases, except He where it is about 8 due to considerably larger atomic density.

On the other hand, the EL yield of excimer EL at these fields significantly exceeds that of NBrS EL predicted theoretically, by about a factor of 100 . In fact, this large difference might be much less, of about a factor of 30 , due to enhanced $\mathrm{NBrS}$ emission observed in experiment (see discussion in
Sect. 5). This difference might be further reduced, because in all noble gases (except Xe) it is necessary to use WLS to record excimer EL, the light losses in which can reach a factor of 10-20 [4].

Thus one may conclude that at moderate reduced electric fields, 1-1.5 Td above the excimer EL threshold, the direct optical readout of two-phase detectors in the visible range, based on NBrS EL, can compete with the readout in the VUV with the use of WLS, based on excimer EL, in all noble gases, except Xe.

Accordingly, NBrS EL is not practical for using in twophase Xe detectors. On the other hand in other noble gases, NBrS EL can be used for the direct (without WLS) optical readout in the visible range, that may serve as a backup solution in two-phase detectors based on $\mathrm{He}, \mathrm{Ne}, \mathrm{Ar}$ and $\mathrm{Kr}$, in case issues with WLS instability over time or non uniformity over large areas should become problematic.

Now let us estimate the electric fields and voltages for a $1 \mathrm{~cm}$ thick EL gap, operated in the two-phase mode in different noble gases: see Table 1 (items 10 and 11). The lighter the noble gas, the lower its boiling temperature $\left(T_{b}\right)$, and the higher its atomic density in the gas phase in the twophase mode: see Table 1 (items 1 and 2). Thereby, the lighter the noble gas, the higher the absolute electric field needed to provide given reduced electric fields. In particular, item 10 of Table 1 presents the electric field strength corresponding to the reduced electric field of $1 \mathrm{Td}$, while item 11 shows the voltage needed to provide the reduced electric field of $10 \mathrm{Td}$ in a $1 \mathrm{~cm}$ thick EL gap. The latter amounts to 237, 34, 8.6, 6.2 and $5.8 \mathrm{kV}$ for $\mathrm{He}, \mathrm{Ne}, \mathrm{Ar}, \mathrm{Kr}$ and Xe respectively.

While for $\mathrm{Ne}, \mathrm{Ar}, \mathrm{Kr}$ and $\mathrm{Xe}$ this voltage is safe with respect to breakdowns, for He it exceeds the breakdown voltage in liquid $\mathrm{He}$, which is about $100 \mathrm{kV}$ [45]. For He, a simple solution to the breakdown problem would be to reduce the EL gap thickness (and thus the gap voltage) by an order of magnitude, down to $1 \mathrm{~mm}$ : the number of photons produced in such a small gap for both NBrS and excimer EL would be still comparable to that for other noble gases: see Table 1 (items 8 and 9). Moreover, for such a small gap thickness the parallel-plate EL gap can be replaced by a more robust thick Gas Electron Multiplier (THGEM, [46]) having a similar thickness and operated in proportional EL mode.

It should be remarked that for two-phase $\mathrm{He}$ and $\mathrm{Ne}$ detectors, the issue of electron emission from the liquid to the gas phase might be another problem, since the electrons in liquid $\mathrm{He}$ and $\mathrm{Ne}$ are trapped in bubbles, in contrast to other noble liquids. This resulted in that penetration of charges from the liquid to the gas phase in two-phase $\mathrm{Ne}$ is more complex and that the trapping time of the electrons at the liquid-gas interface is much larger, than expected [47].

These problems may force to give up of the two-phase mode for He- and Ne-based detectors and lead to the idea of a high-pressure single-phase cryogenic detector with EL 

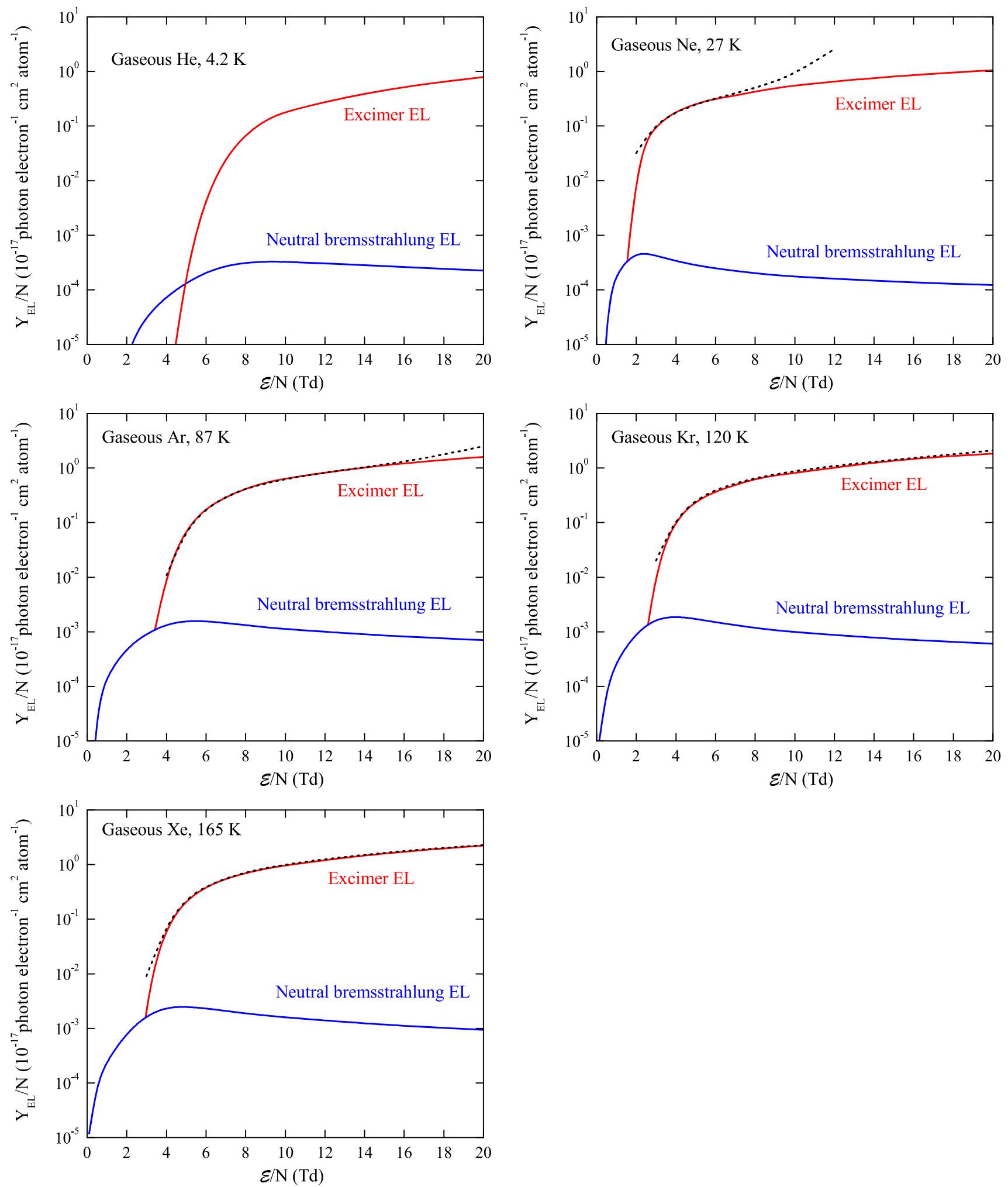

Fig. 12 Reduced EL yield for NBrS EL at $0-1000 \mathrm{~nm}$ and that of excimer EL in noble gases as a function of the reduced electric field, calculated in this work using Boltzmann equation solver (solid lines).

For comparison, the EL yield of excimer EL, calculated using microscopic approach [9], is shown (dashed lines) 


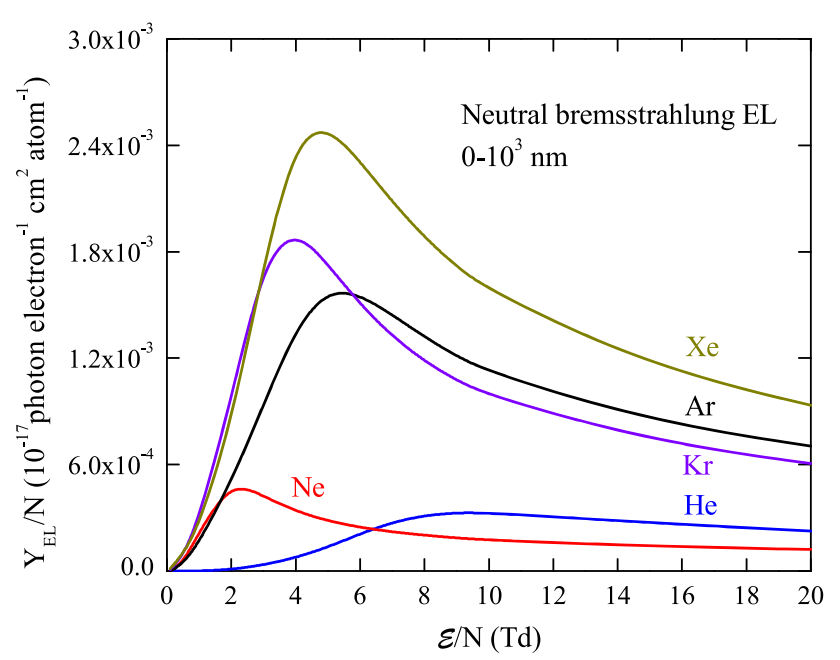

Fig. 13 Reduced EL yield of NBrS EL in noble gases as a function of the reduced electric field, calculated using Eq. (8) integrated over the wavelength range of $0-1000 \mathrm{~nm}$

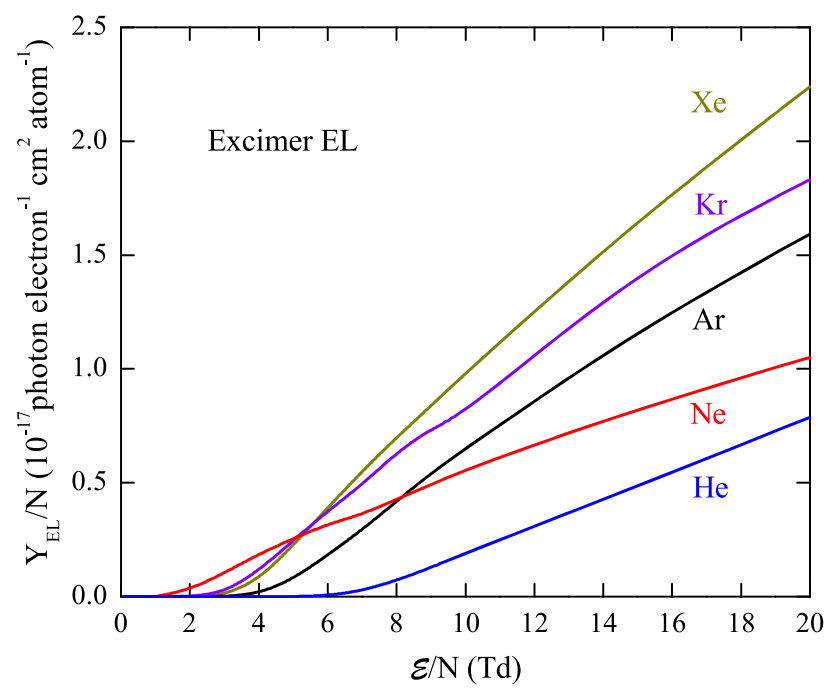

Fig. 14 Reduced EL yield of excimer EL in noble gases as a function of the reduced electric field, calculated using Eq. (11)

gap, operated at temperatures slightly higher than $T_{b}$. Indeed, the gas atomic density at such low temperatures in $\mathrm{Ne}$ and especially in $\mathrm{He}$ can approach to the liquid atomic density in $\mathrm{Ar}, \mathrm{Kr}$, and $\mathrm{Xe}$ : see Table 1 (items 2 and 3), which makes it possible to abandon the liquid phase in $\mathrm{He}$ and $\mathrm{Ne}$ detectors at low temperatures and high $(\leq 10 \mathrm{~atm})$ pressures.

Finally, other prospects to use $\mathrm{He}$ and $\mathrm{Ne}$ media at low temperatures for dark matter and neutrino detectors should also be mentioned [48-51].

\section{Conclusions}

In this work, the electroluminescence (EL) yields and spectra for both neutral bremsstrahlung $(\mathrm{NBrS})$ and excimer EL were calculated in $\mathrm{He}, \mathrm{Ne}, \mathrm{Ar}, \mathrm{Kr}$ and $\mathrm{Xe}$, following the theoretical approach successfully applied to Ar in our previous work [4].

$\mathrm{NBrS}$ EL is predicted to have generally the same properties in all noble gases:

- it has a rather flat emission spectrum extending from the UV to the visible and NIR range, the vast majority of which is above $200 \mathrm{~nm}$;

- it dominates below the excimer EL threshold;

- it can compete with excimer EL in terms of the number of detected photons for moderate reduced electric fields, 1-1.5 Td above the excimer EL threshold;

- it is insignificant compared to excimer EL at higher reduced electric fields.

It was shown that in the two-phase mode, light noble gases $(\mathrm{He}$ and $\mathrm{Ne}$ ) are as good as heavy noble gases ( $\mathrm{Ar}, \mathrm{Kr}$ and $\mathrm{Xe}$ ) in terms of the number of photons (for both $\mathrm{NBrS}$ and excimer EL) emitted in a practical EL gap, of a thickness $1 \mathrm{~mm}$ for $\mathrm{He}$ and $1 \mathrm{~cm}$ for other noble gases.

It was argued that NBrS EL might have practical applications in all noble gases, except Xe: it can be used for the direct (without WLS) optical readout in the visible range, that may serve as a backup solution for two-phase dark matter detectors, in case issues with WLS instabilities should become problematic.

Acknowledgements This work was supported by Russian Science Foundation (project no. 19-12-00008). It was done within the R\&D program of the DarkSide-20k experiment.

Data Availability Statement This manuscript has no associated data or the data will not be deposited. [Authors' comment: The data will be available at any time by contacting the corresponding author.]

Open Access This article is licensed under a Creative Commons Attribution 4.0 International License, which permits use, sharing, adaptation, distribution and reproduction in any medium or format, as long as you give appropriate credit to the original author(s) and the source, provide a link to the Creative Commons licence, and indicate if changes were made. The images or other third party material in this article are included in the article's Creative Commons licence, unless indicated otherwise in a credit line to the material. If material is not included in the article's Creative Commons licence and your intended use is not permitted by statutory regulation or exceeds the permitted use, you will need to obtain permission directly from the copyright holder. To view a copy of this licence, visit http://creativecomm ons.org/licenses/by/4.0/.

Funded by SCOAP ${ }^{3}$. 


\section{References}

1. V. Chepel, H. Araujo, J. Instrum. 8, R04001 (2013). https://doi. org/10.1088/1748-0221/8/04/R04001

2. D. Akimov, A. Bolozdynya, A. Buzulutskov V. Chepel, Two-phase emission detectrors (World Scientific, New Jersey, 2021)

3. A. Buzulutskov, Instruments 4, 16 (2020). https://doi.org/10.3390/ instruments 4020016

4. A. Buzulutskov et al., Astropart. Phys. 103, 29 (2018). https://doi. org/10.1016/j.astropartphys.2018.06.005

5. Y. Butikov et al., Sov. Phys. JETP 30, 24 (1970)

6. A. Bondar et al., Nucl. Instrum. Meth. A 958, 162432 (2020). https://doi.org/10.1016/j.nima.2019.162432

7. C. Monteiro et al., Phys. Lett. B 668, 167 (2008). https://doi.org/ 10.1016/j.physletb.2008.08.030

8. A. Buzulutskov et al., Europhys. Lett. 94, 52001 (2011). https:// doi.org/10.1209/0295-5075/94/52001

9. C. Oliveira et al., Phys. Lett. B 703, 217 (2011). https://doi.org/10. 1016/j.physletb.2011.07.081

10. A. Buzulutskov, Europhys. Lett. 117, 39002 (2017). https://doi. org/10.1209/0295-5075/117/39002

11. N. Schwentner, E. Koch, J. Jortner, Electronic Excitations in Condensed Rare Gases (Springer, Berlin, 1985). https://doi.org/10. 1007/BFb0111641

12. C. Benson et al., Eur. Phys. J. C 78, 329 (2018). https://doi.org/10. 1140/epjc/s10052-018-5807-z

13. E. Aprile, A. Bolotnikov, A. Bolozdynya, T. Doke, Noble Gas Detectors (Wiley, Weinheim, 2006). https://doi.org/10.1002/ 9783527610020

14. R. Huffman et al., Appl. Opt. 4(12), 1581 (1965). https://doi.org/ 10.1364/AO.4.001581

15. A. Morozov et al., J. Appl. Phys. 103, 103301 (2008). https://doi. org/10.1063/1.2931000

16. P. Lindblom, O. Solin, Nucl. Instrum. Meth. A 268, 204 (1988). https://doi.org/10.1016/0168-9002(88)90607-9

17. M. Fraga et al., IEEE Trans. Nucl. Sci. 47, 933 (2000). https://doi. org/10.1109/23.856721

18. C. Aalseth et al., DarkSide-20k Collaboration, Eur. Phys. J. C 81, 153 (2021). https://doi.org/10.1140/epjc/s10052-020-08801-2

19. C. Oliveira et al., Nucl. Instrum. Meth. A 722, 1 (2013). https:// doi.org/10.1016/j.nima.2013.04.061

20. M. Tanaka et al., J. Phys. Conf. Ser. 1468, 012052 (2020). https:// doi.org/10.1088/1742-6596/1468/1/012052

21. M. Kimura et al., J. Instrum. 15(08), C08012 (2020). https://doi. org/10.1088/1748-0221/15/08/C08012

22. T. Takeda et al., J. Phys. Conf. Ser. 1468, 012053 (2020). https:// doi.org/10.1088/1742-6596/1468/1/012053

23. T. Takeda et al., J. Instrum. 15(03), C03007 (2020). https://doi.org/ $10.1088 / 1748-0221 / 15 / 03 / \mathrm{c} 03007$

24. K. Aoyama et al., eprint arXiv:2107.02330 (2021)

25. G. Schulz, Rev. Mod. Phys. 45, 378 (1973). https://doi.org/10. 1103/RevModPhys.45.378
26. L. D’yachkov et al., Sov. Phys. JETP 38, 697 (1974). https://www. osti.gov/biblio/4400199

27. C. Monteiro, Novel approach to Xenon optical TPCs: the presence of Neutral Bremsstrahlung, TIPP Conf., May 24-28, 2021, Vancouver. https://indi.to/m5qnK

28. J. Park et al., Phys. Plasmas 7(8), 3141 (2000). https://doi.org/10. $1063 / 1.874220$

29. O. Firsov, M. Chibisov, Sov. Phys. JETP 12, 1235 (1961). https:// www.osti.gov/biblio/4094108

30. V. Kas'yanov, A. Starostin, Sov. Phys. JETP 21, 193 (1965)

31. A. Dalgarno, N. Lane, Astrophys. J. 145, 623 (1966). https://doi. org/10.1086/148801

32. L. Biberman, G. Norman, Sov. Phys. Uspekhi 10, 52 (1967). https:// doi.org/10.1070/pu1967v010n01abeh003199

33. G. Hagelaar, L. Pitchford, Plasma Sources Sci. Technol. 14, 722 (2005). https://doi.org/10.1088/0963-0252/14/4/011

34. https://fr.lxcat.net/solvers/bolsigplus/

35. http://www.lxcat.net/biagi

36. S. Biagi, Nucl. Instrum. Meth. A 421(1), 234 (1999). https://doi. org/10.1016/S0168-9002(98)01233-9

37. http://www.lxcat.net/bsr

38. A. Peisert, F. Sauli, Drift and Diffusion of Electrons in Gases: A Compilation. CERN Yellow Reports: Monographs (CERN, Geneva, 1984). https://doi.org/10.5170/CERN-1984-008

39. V. Kas'yanov, A. Starostin, Sov. J. Plasma Phys. 4, 67 (1978). https://www.osti.gov/biblio/6666613

40. V. Fastovsky, A. Rovinsky, Y. Petrovsky, Inert Gases (Atomizdat, Moscow, 1971) (in Russian)

41. F. Theeuwes, R. Bearman, J. Chem. Thermodyn. 2, 507 (1970). https://doi.org/10.1016/0021-9614(70)90100-X

42. R. Bernabei et al., Int. J. Mod. Phys. A 30, 1530053 (2015). https:// doi.org/10.1142/S0217751X15300537

43. E. Aprile et al., Astropart. Phys. 35, 573 (2012). https://doi.org/10. 1016/j.astropartphys.2012.01.003

44. P. Agnes et al., Phys. Lett. B 743, 456 (2015). https://doi.org/10. 1016/j.physletb.2015.03.012

45. J. Gerhold et al., Cryogenics 34, 579 (1994). https://doi.org/10 1016/0011-2275(94)90183-X

46. A. Breskin et al., Nucl. Instrum. Meth. A 598, 107 (2009). https:// doi.org/10.1016/j.nima.2008.08.062

47. R. Galea et al., JINST 2, P04007 (2007). https://doi.org/10.1088/ 1748-0221/2/04/P04007

48. A. Buzulutskov et al., Nucl. Instrum. Meth. A 548 (2005). https:// doi.org/10.1016/j.nima.2005.04.066

49. Y. Ju et al., Cryogenics 47, 81 (2007). https://doi.org/10.1016/j. cryogenics.2006.08.008

50. W. Guo, D. Mckinsey, Phys. Rev. D 87, 115001 (2013). https://doi. org/10.1103/PhysRevD.87.115001

51. J. Liao et al., eprint arXiv:2103.02161 (2021) 